\title{
Magnetospheric lion roars
}

\author{
W. Baumjohann ${ }^{1}$, E. Georgescu ${ }^{1,2}$, K.-H. Fornacon ${ }^{3}$, H. U. Auster ${ }^{3}$, R. A. Treumann ${ }^{1}$, G. Haerendel ${ }^{1}$ \\ ${ }^{1}$ Max-Planck-Institut für extraterrestrische Physik, Garching, Germany \\ ${ }^{2}$ Institut for Space Sciences, Bucharest, Romania \\ ${ }^{3}$ Institut für Geophysik und Meteorologie, TU Braunschweig, Germany
}

Received: 6 September 1999 / Revised: 6 December 1999 / Accepted: 7 January 2000

\begin{abstract}
The Equator-S magnetometer is very sensitive and has a sampling rate normally of $128 \mathrm{~Hz}$. The high sampling rate for the first time allows detection of ELF waves between the ion cyclotron and the lower hybrid frequencies in the equatorial dawnside magnetosphere. The characteristics of these waves are virtually identical to the lion roars typically seen at the bottom of the magnetic troughs of magnetosheath mirror waves. The magnetospheric lion roars are near-monochromatic packets of electron whistler waves lasting for a few wave cycles only, typically $0.2 \mathrm{~s}$. They are right-hand circularly polarized waves with typical amplitudes of $0.5 \mathrm{nT}$ at around one tenth of the electron gyrofrequency. The cone angle between wave vector and ambient field is nearly always smaller than $1^{\circ}$.
\end{abstract}

Key words: Magnetospheric physics (magnetospheric configuration and dynamics; MHD waves and instabilities; plasma waves and instabilities)

\section{Introduction}

More than 20 years ago, Smith and Tsurutani (1976) published the classic paper on detailed search-coil magnetometer observations of lion roars inside magnetic troughs in the dayside magnetosheath. They found that the lion roars are narrow-banded right-hand polarized waves, basically electron cyclotron waves, that are relatively short-lived, about $2 \mathrm{~s}$ and that they have typical frequencies of about $120 \mathrm{~Hz}$. Some years later, Tsurutani et al. (1982) studied the same phenomenon with the plasma wave instrument on ISEE and found these waves at somewhat lower frequencies (50$100 \mathrm{~Hz}$ ). More recently, Zhang et al. (1998) used Geotail waveform capture measurements and found magnetosheath lion roars between $20-300 \mathrm{~Hz}$ and

Correspondence to: W. Baumjohann
Baumjohann et al. (1999) studied the waveform of magnetosheath lion roars below $64 \mathrm{~Hz}$, using the fluxgate magnetometer on board Equator-S.

However, until very recently, this type of electron whistler waves had not been observed inside the Earth's magnetosphere. Pickett et al. (1999) were the first to observe a case of narrow band whistler mode waves in the cusp region with the Polar satellite. These waves had frequencies near $200-300 \mathrm{~Hz}$, had a clear packet structure and resembled lion roars. Using the Equator-S fluxgate magnetometer, we searched for these waves in the equatorial plane of the dawnside magnetosphere and found that they are abundant whenever the background magnetic field is low enough. In the following we will show that the characteristics of these electron whistlers resemble closely those obtained by Baumjohann et al. (1999) for the magnetosheath lion roars and will conclude that lion roars are not only abundant in the magnetosheath, but also in the magnetosphere, at least the dawnside equatorial outer region.

\section{Instrumentation}

The Equator-S magnetic field instrument is fully described in Fornacon et al. (1999). In short, it consists of two units with a pair of three-axes fluxgate magnetometers each. The sensors of the primary and the redundant units are mounted on two rigid booms, with the main sensor located at the end of the $1.8-\mathrm{m}$ boom and the other $50 \mathrm{~cm}$ further inboard. During the interval used in the present study, the sampling rate was 128 vectors/s, at a resolution of about $10 \mathrm{pT}$.

\section{Data}

Before proceeding to the full data set, we first present some typical examples. Figure 1 shows the waveform (high-pass filtered, $f \geq 4 \mathrm{~Hz}$, component perpendicular to the main field) of a magnetospheric electron whistler observed on 11 April, 1998. Only one component is 


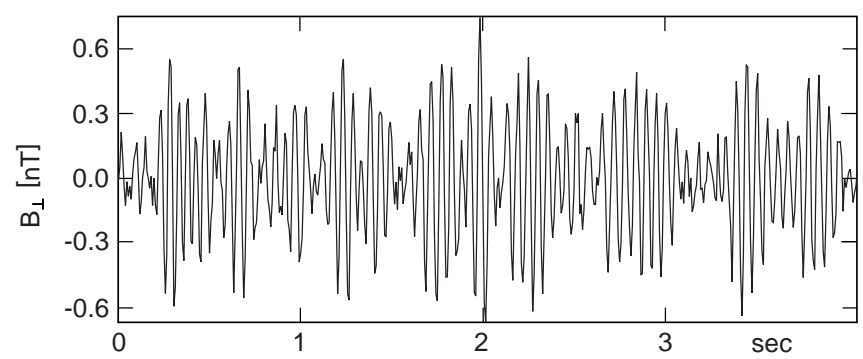

Fig. 1. Lion roar waveform perpendicular to the mean field
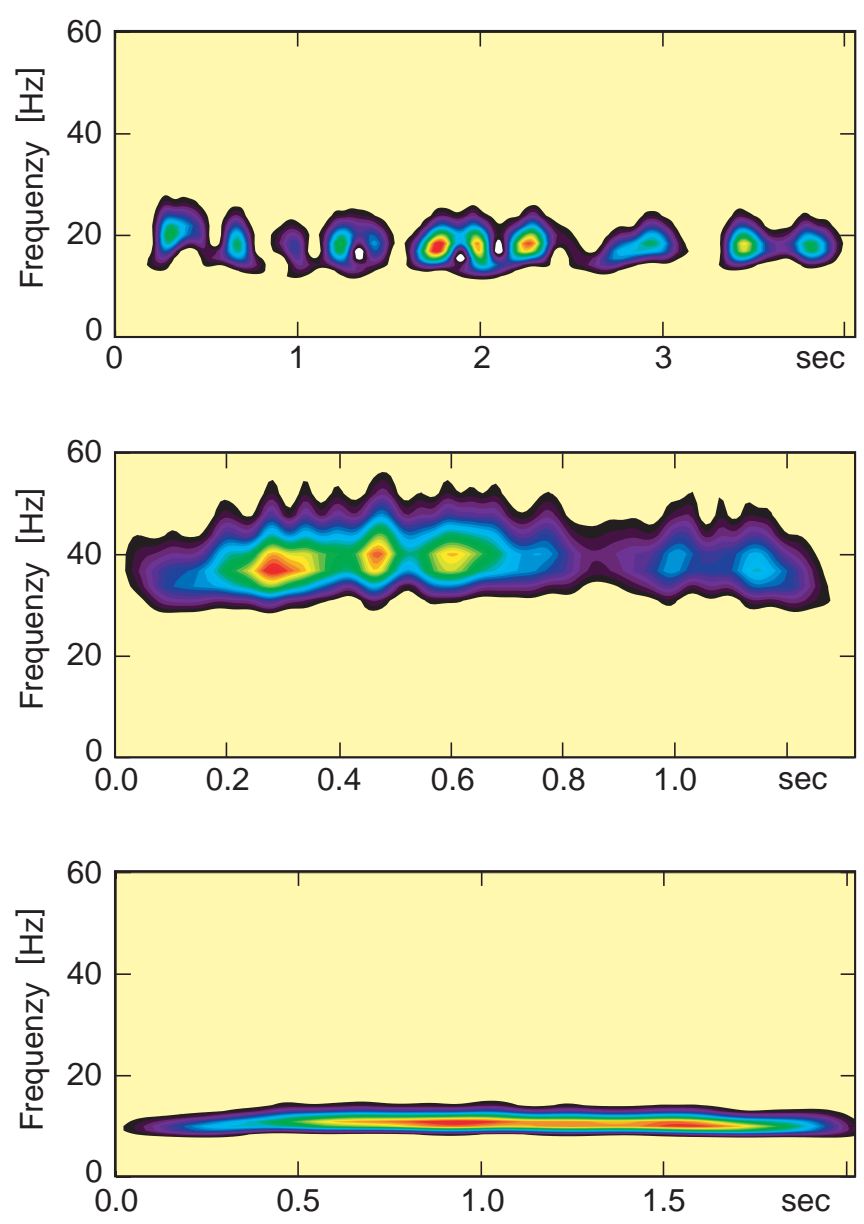

Fig. 2. Right-hand polarized wavelet spectrograms for three magnetospheric lion roar events

shown, but the other transverse component is nearly identical, just phase-shifted by $90^{\circ}$, indicating right-hand circular polarization of the wave.

Since the lion roar signals are rather short-lived, we use a wavelet analysis to determine spectrograms of the signals. The wavelet used is the Morlet wavelet, which is best suited for this type of analysis (Lui and Najmi, 1997; Torrence and Compo, 1998). By transforming the complex signal $\left(\mathbf{B}_{r, l}=\mathbf{B}_{x} \pm i \mathbf{B}_{y}\right)$, we calculate the righthand and left-hand polarization spectrograms. Since the signals are all right-hand circularly polarized, Fig. 2 shows the spectrogram of the right-hand polarized signal of Fig. 1 (upper panel) plus two more examples observed on 22 April (middle panel) and 11 April, 1998 (lower panel).

Figure 1 and the upper panel of Fig. 2 show a rather long-lived signal, about 80 wave cycles with an amplitude of up to $\pm 0.6 \mathrm{nT}$. The signal is nearly monochromatic with a center frequency near $20 \mathrm{~Hz}$. A packet structure is clearly visible. The signal in the middle panel of Fig. 2 is centered around a frequency of about $35 \mathrm{~Hz}$ and also exhibits packet structure. That the electron whistlers can be observed down to about $10 \mathrm{~Hz}$ and may come in longer packets is visible in the lower panel of Fig. 2.

Since by visual inspection we found that electron whistlers were often observed by Equator-S in the dawnside magnetosphere, whenever the background field was weak, we automatically scanned all our data obtained in the 02-06 magnetic local time (MLT) sector of the magnetosphere, whenever the ambient field strength was below $20 \mathrm{nT}$. The result is that about $25 \%$ of these periods contained right-handed circularly polarized waves between 8 and $64 \mathrm{~Hz}$, which we analyze in this section. In particular we look at the frequency and its dependence on the mirror trough background field, the duration of the lion roar wave packets, and the cone angle of the wave vector with the ambient field.

But before going to the characteristics of the waves, let us first look where they occur. Figure 3 clearly shows that there is no preferred region, neither radial distance (for $R \geq 8 R_{E}$ ), nor magnetic local time or distance from the model neutral sheet of $\mathrm{Zhu}$ and $\mathrm{Xu}$ (1994). The waves are evenly distributed in the dawnside equatorial magnetosphere, wherever the ambient field is low. As can be seen in the upper panel of Fig. 4, the waves are often found inside magnetic troughs which resemble the magnetic troughs of magnetosheath mirror modes. However, as evident from the lower panel of this figure, they can also occur outside ambient magnetic field depletions, like the type-B lion roars of Zhang et al. (1998).

Figure 5 shows the occurrence distribution of the lion roar frequencies, normalized to the electron gyrofrequency, $f_{c e}$, for the more than 27000 wave packets found and analyzed. The normalized frequencies are peaked near $0.1 f_{c e}$, with most of the values in the range $0.05 \leq f / f_{c e} \leq 0.15$. Case distribution as well as average value are similar to those found by Zhang et al. (1998) and nearly identical to those presented by Baumjohann et al. (1999), both for magnetosheath lion roars.

The packet structure of the waves visible in most of the cases presented is also found in the statistical analysis. Figure 6 gives the occurrence rates of the duration of the lion roar wave packets in number of wave cycles. Close to two thirds of all cases have a wave packet length of less than five wave cycles and about $90 \%$ last less than ten wave cycles, very similar to the results of Baumjohann et al. (1999) for the magnetosheath lion roars.

We have also determined the wave normal direction as the direction of the minimum variance, i.e., along the eigenvector having the smallest eigenvalue in a mini- 

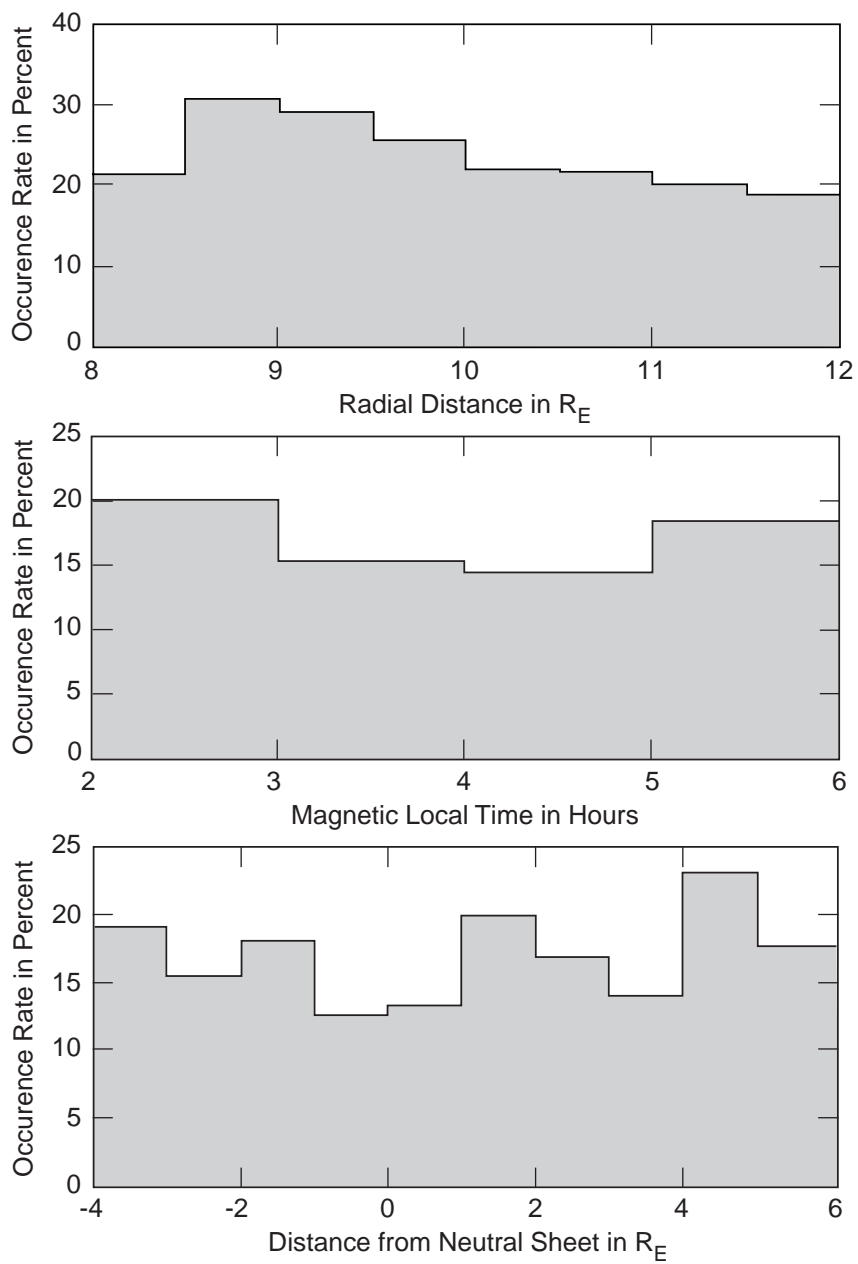

Fig. 3. Occurrence rate of electron whistlers for $|\mathbf{B}|<20 \mathrm{nT}$ with respect to radial distance (upper panel), magnetic local time (middle panel), and distance from a model neutral sheet (lower panel)
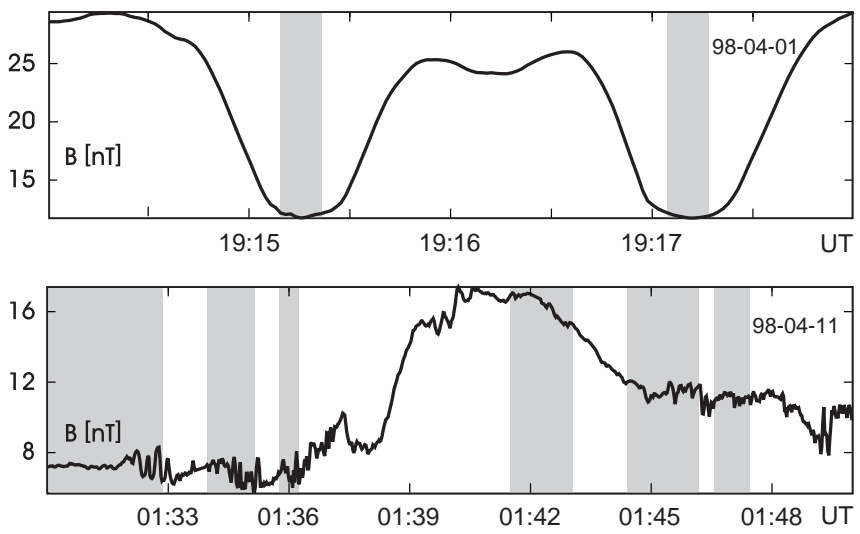

Fig. 4. Ambient magnetic field strength and occurrence of electron whistlers in the shaded intervals

mum variance analysis (see, e.g., Smith and Tsurutani, 1976; Zhang et al. (1998). The wave normal direction, i.e., its k-vector, was clearly aligned with the background magnetic field. The occurrence distribution of

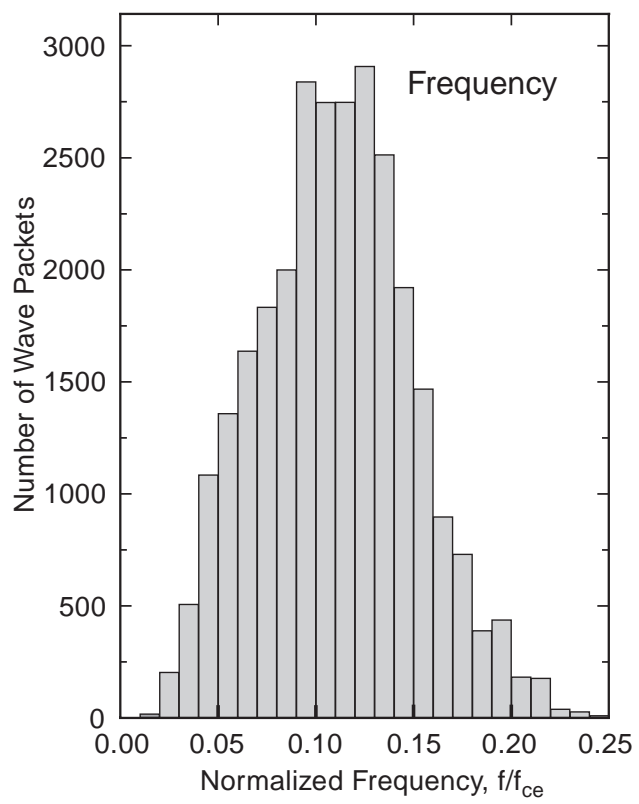

Fig. 5. Occurrence distribution of normalized lion roar frequencies

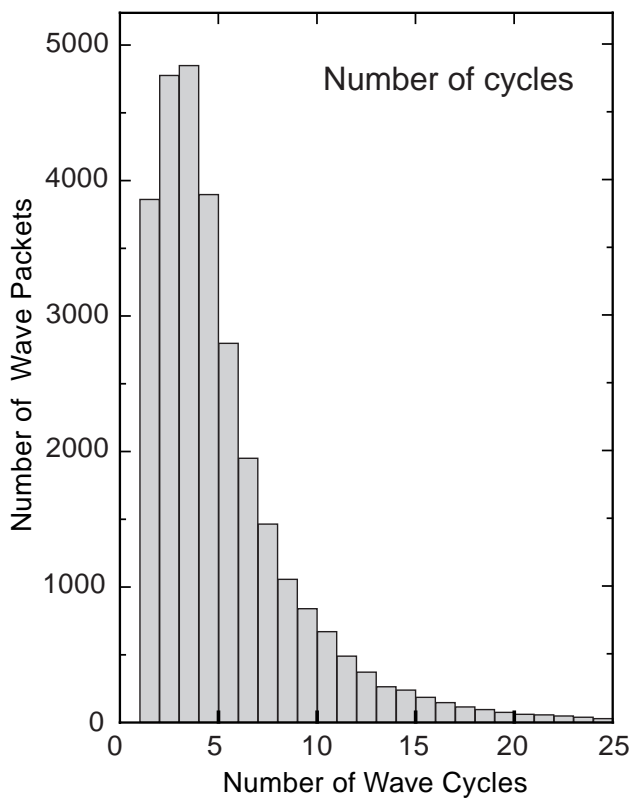

Fig. 6. Occurrence distribution of lion roar wave packet duration

the cone angle, $\theta_{k b}$, is given in Fig. 7. Except for about 50 cases, all cone angles are $<1^{\circ}$. In fact, the cone angle distribution drops off sharply beyond, say, $0.2^{\circ}$.

These values are again virtually identical to those found by Baumjohann et al. (1999). That they are much smaller than the typical cone angles of $10-30^{\circ}$ found by Zhang et al. (1998) and Smith and Tsurutani (1976) has already been explained in Baumjohann et al. (1999): We suspect that the authors of the earlier papers on lion roars have used too long stretches of data for their minimum variance analysis and, thus, have not taken into account the temporal variability of the ambient field, even on the scale of seconds. 


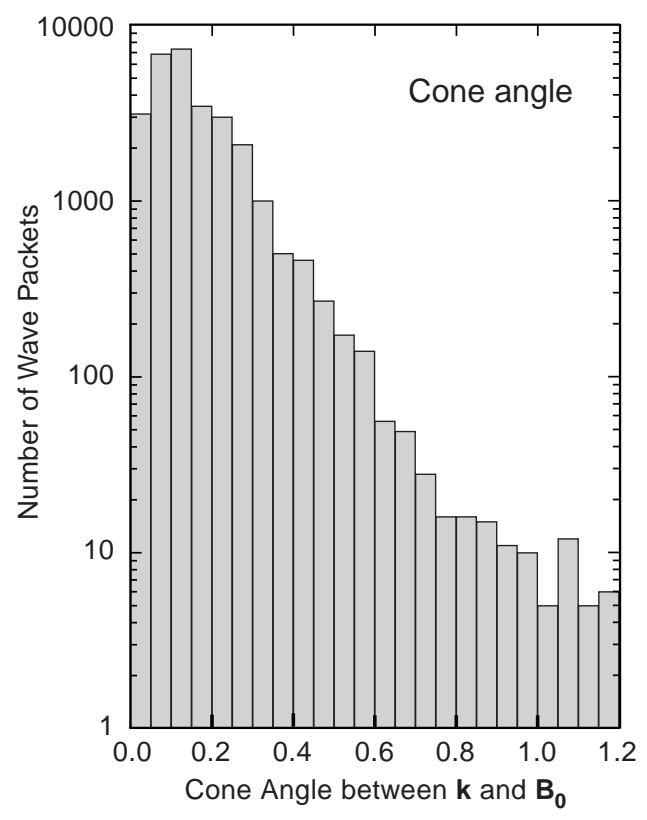

Fig. 7. Occurrence distribution of cone angle between lion roar wave vector and ambient magnetic field

Table 1. Average frequency, wave packet duration, and wave cone angle for magnetospheric and magnetosheath electron whistlers

\begin{tabular}{lll}
\hline Quantity & Magnetosphere & Magnetosheath \\
\hline Centre frequency & $f / f_{c e}=0.10$ & $f / f_{c e}=0.09$ \\
Packet duration & $\Delta t=0.19 \mathrm{~s}$ & $\Delta t=0.25 \mathrm{~s}$ \\
Wave cone angle & $\theta_{k B}=0.15^{\circ}$ & $\theta_{k B}=0.33^{\circ}$ \\
\hline
\end{tabular}

\section{Summary}

The present study shows that the characteristics of electron whistlers in the dawnside equatorial magnetosphere, i.e., (1) near-monochromatic right-hand circularly polarized, (2) typical frequencies of $0.1 f_{c e}$, (3) packet structure with often less than 5 wave cycles, (4) wave cone angles of less than $1^{\circ}$, are virtually identical to those of magnetosheath lion roars. A comparison of average numbers for frequency, packet duration, and cone angle, $\theta_{k B}$, for magnetospheric and magnetosheath electron whistlers are given in Table 1, with the latter values taken from Baumjohann et al. (1999).

\section{Conclusion}

Since the Equator-S electron plasma instrument failed and the ion data is not yet available with an accuracy needed, we cannot draw any new conclusions on the nature of these waves other than those discussed in Baumjohann et al. (1999) at this stage.

The whistler waves found in the dawnside equatorial magnetosphere have the same characteristics as those found in the same frequency range by Baumjohann et al. (1999) in the magnetosheath. We believe that they can rightfully be called magnetospheric lion roars. As first shown by Tsurutani et al. (1982), magnetosheath lion roars are caused by a perpendicular electron pressure anisotropy in the magnetic troughs of mirror mode-like structures. Such electron pressure anisotropies have been inferred by Baumjohann et al. (1999) from lion roar observations in the magnetosheath. There the typical resonant electron energies have been explicitly determined. As the present observations fall into the same frequency band and the bandwidths are similar, we may conclude that it is the same electron energy range that is responsible for the excitation of the magnetospheric lion roars. Whenever mirror effects cause depressions in the magnetic field, trapped electrons can excite whistler mode waves in the corresponding frequency range.

The modes that occur outside of magnetic troughs, i.e. those indicated in the lower panel of Fig. 4 and the type-B lion roars of Zhang et al. (1998), may have been excited in a similar way and have travelled along the ambient magnetic field to the observation point. Unfortunately, the lack of plasma data does not allow for a check of this assumption. However, since whistler waves propagate at a fraction of the electron Alfvén speed, which for the outer magnetosphere typically is of the order of $10^{4} \mathrm{~km} / \mathrm{s}$, they can leak from their low-field excitation region and propagate to another observation site.

It is of course not unknown that whistlers can be excited in the outer magnetosphere. The most typical examples are the equatorial chorus emissions frequently observed during substorms (Tsurutani and Smith, 1974) and associated with tail plasma injections. The newly injected plasma destabilizes the whistler mode in a relatively broad frequency band $(50-100 \mathrm{~Hz}$ wide), in contrast to our observations of a very narrow band.

Most of the whistlers forming the lion roars discussed here are generated locally in small-scale decreases of the magnetospheric magnetic field. The narrow-bandedness indicates that they are caused by a small anisotropy of the electrons trapped in these mirror-like structures. Thus they seem clear relatives of the magnetosheath lion roars (Baumjohann et al. 1999). The much lower number of whistlers we see at magnetic field gradients may likely be lion roars that have travelled there from neighbouring troughs, because they are also narrowbanded. But we cannot exclude the possibility that at least some of them are related to chorus-like emissions.

Acknowledgements. Equator-S was financially supported via grant 50 OC 94024 by the German Space Agency, DARA (now DLR). The wavelet software was provided by C. Torrence and G. Compo, and is available at http://paos.colorado.edu/research/wavelets/.

Topical Editor G. Chanteur thanks N. Cornilleau-Wehrlin and another referee for their help in evaluating this paper.

\section{References}

Baumjohann, W., R. A. Treumann, E. Georgescu, G. Haerendel, K.-H. Fornacon, and H. U. Auster, Waveform and packet structure of lion roars, Ann. Geophysicae, 17, 1528-1534, 1999. 
Fornacon, K.-H., H. U. Auster, E. Georgescu, W. Baumjohann, K.-H. Glaßmeier, J. Rustenbach, and M. Dunlop, The magnetic field experiment onboard Equator-S and its scientific possibilities, Ann. Geophysicae, 17, 1521-1527, 1999.

Lui, A. T. Y., and A.-H. Najmi, Time-frequency decomposition of signals in a current disruption event, Geophys. Res. Lett., 24, 3157-3160, 1997.

Pickett, J. S., et al., Plasma waves observed during cusp energetic particle events and their correlation with Polar and Akebono satellite and ground data, Adv. Space Res., 24(1), 23-33, 1999.

Smith, E. J., and B. T. Tsurutani, Magnetosheath lion roars, J. Geophys. Res., 81, 2261-2266, 1976.

Torrence, C., and G. P. Compo, A practical guide to wavelet analysis, Bull. Am. Meteorol. Soc., 79, 61-78, 1998.
Tsurutani, B. T., and E. J. Smith, Postmidnight chorus: a substorm phenomenon, J. Geophys. Res., 79, 118-127, 1974.

Tsurutani, B. T., E. J. Smith, R. R. Anderson, K. W. Ogilvie, J. D. Scudder, D. N. Baker, and S. J. Bame, Lion roars and nonoscillatory drift mirror waves in the magnetosheath, J. Geophys. Res., 87, 6060-6072, 1982.

Zhang, Y., H. Matsumoto, and H. Kojima, Lion roars in the magnetosheath: the Geotail observations, J. Geophys. Res., 103, 4615-4626, 1998.

Zhu, M., and R.-L. Xu, A continuous neutral sheet model and a normal curved coordinate system in the magnetotail, Chin. J. Space Sci., 14, 269-278, 1994. 